\title{
Circulación del Golfo de Arauco en un período de transición estacional: Un nuevo enfoque
}

\author{
Carolina E. Parada V. ${ }^{1}$, Marcus A. Sobarzo B. ${ }^{2}$, Dante Figueroa M. ${ }^{3}$ y Leonardo Castro C. ${ }^{1}$ \\ ${ }^{1}$ Departamento de Oceanografía, Facultad de Ciencias Naturales y Oceanográficas \\ Universidad de Concepción, Casilla 160-C, Concepción, Chile \\ ${ }^{2}$ Centro EULA-Chile, Universidad de Concepción, Casilla 160-C, Concepción, Chile \\ ${ }^{3}$ Departamento de Física de la Atmósfera y el Océano, Universidad de Concepción \\ Casilla 160-C, Concepción, Chile
}

Recibido: 22 agosto 1999; versión corregida: 22 septiembre 2000; aceptado: 4 noviembre 2000

\begin{abstract}
RESUMEN. En este estudio se caracterizaron los patrones de circulación en el interior del Golfo de Arauco ( $37^{\circ} 10^{\prime} \mathrm{S}$ - 36 45'S, Chile) que están asociados a períodos de intensificación y relajación de la surgencia costera durante un período de transición verano-otoño. Se analizaron imágenes satelitales de TSM (10, 16, 22 y 27 de marzo), series de corrientes (a 10 y $28 \mathrm{~m}$ ) provenientes de tres fondeos de correntómetros y series de viento. Los resultados muestran que la marea semidiurna, las fluctuaciones diurnas del viento S-SW y los procesos de surgencia y de hundimiento en la plataforma adyacente al Golfo, son factores importantes en la determinación de la circulación al interior de éste. La discusión se centró en fluctuaciones del orden de tres a diez días identificándose, durante el período de estudio, 4 eventos de relajación (9-13, 19-20, 23-24, y 30-31 de marzo) y 4 de surgencia (2-8, 14-18, 21-22 y 25-29 de marzo) adyacentes al Golfo. El viento S-SW, favorable a surgencia, forzó una circulación antihoraria de una sola capa durante el máximo desarrollo de la surgencia. Durante viento N, en cambio, la circulación mostró una tendencia horaria durante procesos de hundimiento fuera del Golfo. En períodos de transición o debilitamiento del viento, ya sea de viento SSW o N, la circulación mostró un sistema de doble capa: superficial antihoraria y subsuperficial horaria. Se propone un esquema conceptual de la circulación del Golfo de Arauco en períodos de Surgencia-Relajación basado en el patrón de circulación subinercial. Este patrón dependió, principalmente, de las fluctuaciones de la intensidad y dirección del viento y de la entrada de agua hacia el interior del Golfo a través de su "Boca Grande".
\end{abstract}

Palabras claves: surgencia, viento, TSM, circulación costera, Golfo de Arauco, Chile.

\section{Circulation of the Arauco Gulf in a seasonal transitional period: A new approach}

\begin{abstract}
This study characterizes the circulation patterns in the Arauco Gulf $\left(37^{\circ} 10^{\prime} \mathrm{S}-36^{\circ} 45^{\prime} \mathrm{S}\right.$, Chile) which are associated to the intensification and relaxation of the coastal upwelling, during a summer-autumn transition period. Sea Surface Temperature images (10, 16, 22 and 27 of March), current time series (10 and $28 \mathrm{~m}$ depth) from three current-meter moorings, and wind time series were analyzed. The results indicated that semidiurnal tides, diurnal fluctuations of S-SW wind, upwelling and downwelling in the shelf adjacent to the Gulf are important factors that determine the circulation in the Arauco Gulf. The discussion was centered in the 3-10 day oscillations. Four relaxation events (9-13, 19-20, 23-24, and 30-31 of March) and four upwelling events (2-8, 14-18, 21-22 and 25-29 of March) adjacent to the Gulf were identified during the study period. S-SW wind forced a one-layer anticlockwise circulation during the most intense upwelling. In contrast, during $\mathrm{N}$ wind, the circulation in the Gulf showed a clockwise trend, associated to downwelling in the region adjacent to the Gulf. In transitional periods or during weak N or S-SW winds, the circulation showed a double layer system: an anticlockwise trend near the surface and a clockwise trend in the subsurface. A conceptual scheme of circulation in the Arauco Gulf is suggested for upwelling-relaxation periods, based in the sub-inertial circulation pattern, and depending mainly on the fluctuations of the wind strength and direction, and on the inflow of water into the Gulf through the "Boca Grande", its main connection with the open sea.
\end{abstract}

Key words: upwelling, wind, SST, coastal circulation, Arauco Gulf, Chile. 


\section{INTRODUCCIÓN}

La zona costera frente a la VIII Región es uno de los centros pesqueros pelágicos más importantes en Chile (Yáñez et al., 1996). En esta zona se ubica el Golfo de Arauco ( $36^{\circ} 45^{\prime}$ S hasta $37^{\circ} 10^{\prime}$ S, Fig. 1), área semicerrada importante por representar un centro de desove y de retención de larvas de especies pelágicas (Arcos et al., 1987; Djurfeldt, 1989; Castillo et al., 1991; Arcos et al., 1996; Castro et al., 1997). Debido a esto, es determinante el conocimiento de las características oceanográficas y dinámicas del Golfo de Arauco para entender las variaciones en la sobrevivencia de los estados tempranos de desarrollo de algunas especies de peces. Sin embargo, el conocimiento actual sólo incluye algunos aspectos de la hidrografía del Golfo (Alarcón, 1970; Ahumada y Chuecas, 1979; Arcos y Salamanca, 1984; Sobarzo, 1993; Sobarzo et al., 1993; Urrutia, 1993; Sobarzo, 1994;), su relación con la meteorología local (Saavedra, 1980; Ruiz, 1994; Herrera,
1994; Picarte et al., 1996) y, parcialmente, aspectos dinámicos relativos a la circulación costera (Bernal et al., 1986; Djurfeldt, 1989) y a la pluma del río Biobío (Urrutia, 1993). El objetivo de este estudio es caracterizar los patrones de circulación en el interior del Golfo de Arauco que están asociados a períodos de intensificación y relajación de la surgencia costera durante un período de transición verano-otoño, a través de mediciones simultáneas de corrientes marinas, viento superficial, temperatura superficial del mar.

\section{MÉTODOS}

\section{Recopilación de Datos}

La información de viento analizada correspondió a una serie de tiempo horaria de magnitud y dirección para el período de marzo de 1996 registrada por la Estación Carriel Sur, Concepción (36²46'S;

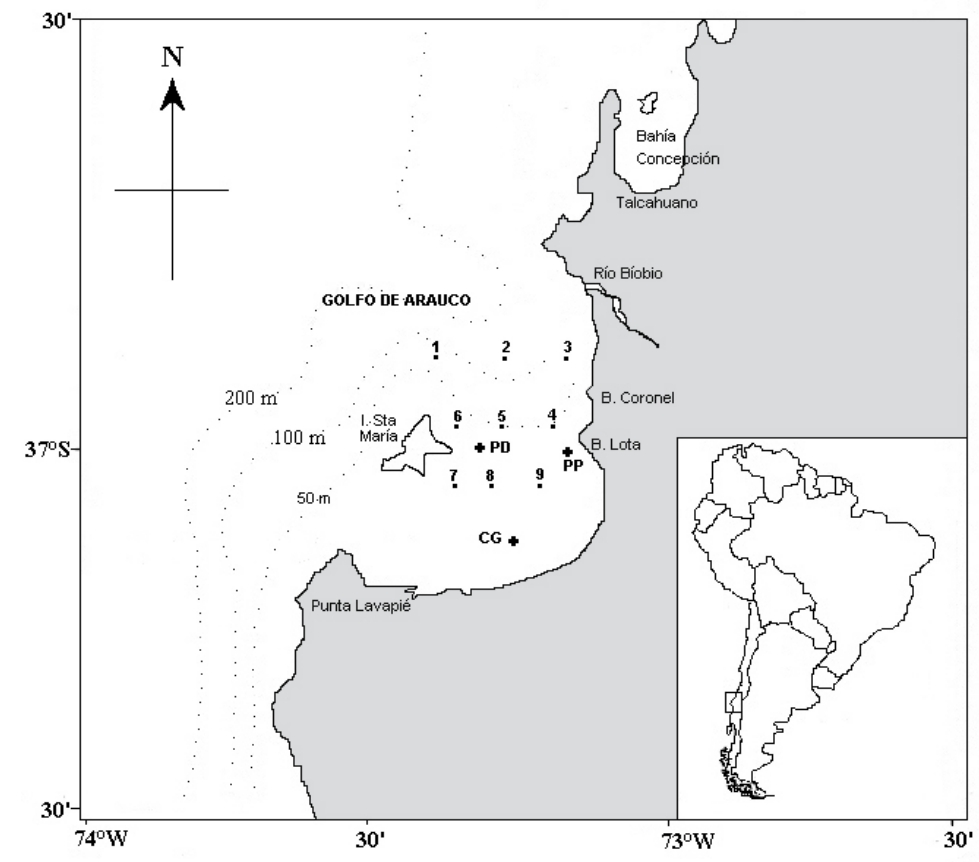

Figura 1. Mapa del Golfo de Arauco mostrando las 9 estaciones oceanográficas y la ubicación de los correntómetros fondeados en Punta Delicada (PD) a 10 y 28 m, Punta Puchoco (PP) a 10 y 28 m y Cabeza del Golfo (CG) a 25 m.

Figure 1. Map of the Arauco Gulf showing the location of the nine oceanographic stations and the current meter in Punta Delicada (PD) at 10 and $28 \mathrm{~m}$ depth, Punta Puchoco (PP) at 10 and $28 \mathrm{~m}$ depth, and Cabeza del Golfo (CG) at $25 \mathrm{~m}$ depth. 
$\left.73^{\circ} 04^{\prime} \mathrm{W}\right)$. Se analizaron las series horarias de magnitud y dirección de las corrientes marinas, para el período marzo de 1996, provenientes de 5 correntómetros Sensor Data ubicados en tres fondeos: Punta Delicada (PD), Punta Puchoco (PP) y Cabeza del Golfo (CG), e instalados a 10 y $28 \mathrm{~m}$ de profundidad en PD y PP, y a $25 \mathrm{~m}$ de profundidad en CG (Fig.1). Se asume que los correntómetros ubicados en PD, PP y CG representan el comportamiento de la corriente al sur y al norte de la "Boca Grande" y en la "Cabeza del Golfo", respectivamente. Se realizaron cinco cruceros oceanográficos durante los días 5, 9, 14, 22 y 27 de marzo de 1996 en 9 estaciones ubicadas en el Golfo de Arauco, las que se distribuyeron en transectas con 3 estaciones cada una (Fig. 1). Las imágenes satelitales de temperatura superficial del mar (TSM) provienen de los días 10, 16, 22 y 27 de marzo de 1996.

\section{Análisis espectral de las series de viento y corriente}

Se separaron las frecuencias mareales y subinerciales de las series de corriente y de viento, mediante el filtro coseno Lanczos simétrico de 121 pesos y con frecuencia de corte de 0,6 cpd (Walter y Heston, 1982; Thompson, 1983). Se realizó un análisis espectral de las series, por componente, para identificar el armónico típico de la brisa marina y de la marea. Las series fueron separadas en segmentos menores para aumentar los grados de libertad. El análisis se realizó utilizando la Transformada Rápida de Fourier, método que separa una señal en sus constituyentes sinusoidales con coeficientes aleatorios no correlacionados. A las series se les extrajo la media y la tendencia lineal. Luego se le aplicó una ventana coseno (coseno taper 15\%) a ambos extremos de cada serie, lo que reduce las fluctuaciones ficticias producidas por la transformada de Fourier en los extremos de la serie (Pizarro, 1991). La estimación espectral está determinada por una distribución $\mathrm{X}^{2}$ con 6 grados de libertad. El intervalo de confianza para los estimadores espectrales utiliza una escala logarítmica en la ordenada (Jenkins y Watts, 1969; Pizarro, 1991):

$$
\log \frac{v}{X_{v}(1-\alpha / 2)}, \log \frac{v}{X_{v}(\alpha / 2)}
$$

donde $v$ son los grados de libertad, $\alpha$ es el nivel de significancia, y $X_{v}(1-\alpha / 2)$ y $X_{v}(\alpha / 2)$ es la probabilidad acumulada para $(1-\alpha / 2)$ y para $(\alpha / 2)$, respectivamente.

\section{Intensidad del viento y caracterización de eventos de surgencia en Golfo de Arauco}

Se identificaron los períodos de surgencia mediante la estimación del índice de Bakun (Bakun, 1978) y el análisis de las imágenes satelitales. La intensidad del viento fue diagramada a través de un gráfico de trazos y el índice de surgencia diario $I_{s}$ (Bakun, 1978) se estimó a partir del transporte Ekman, $M$, por cada 100 m de línea de costa

$$
\mathrm{I}_{\mathrm{s}}=\frac{\mathrm{M}}{\rho_{\mathrm{a}}} * 100
$$

donde $\rho_{a}$ es la densidad del agua en $\mathrm{kg} / \mathrm{m}^{3}, M$ es transporte Ekman definido como el cuociente entre el esfuerzo del viento, $\tau$, y el parámetro de Coriolis, $f$.

$$
\mathrm{M}=\frac{\tau}{f}
$$

El esfuerzo del viento se estimó en función de un coeficiente de arrastre dependiente de la velocidad del viento (Wu, 1982).

$$
\tau=\mathrm{d}_{\mathrm{a}} \mathrm{C}_{10} * \neq \mathrm{V} \neq * \mathrm{~V}
$$

donde $\mathrm{V}$ es la componente norte-sur de la intensidad del viento, $\mathrm{d}_{\mathrm{a}}$ es la densidad del aire $\left(1,24 \mathrm{~kg} / \mathrm{m}^{-3}\right), \mathrm{y}$ $\mathrm{C}_{10}$ es el coeficiente de arrastre del viento dado por (Wu, 1982):

$$
\mathrm{C}_{10}=\left(0.8+0.065 * \mathrm{~V}_{10}\right) \times 10^{-3}
$$

donde $\mathrm{V}_{10}$ es la intensidad del viento en $\mathrm{ms}^{-1}$ medido a $10 \mathrm{~m}$ sobre el nivel del mar.

\section{Análisis direccional de la corriente residual}

Se realizó un análisis direccional de la corriente residual a través del método del Diagrama del Vector Progresivo (Krause, 1984). Las series de corriente filtrada o residual se graficaron para todo el período analizándose la relación entre los eventos de surgencia dados por el índice de Bakun, las TSM y la entrada o salida de agua desde el Golfo de Arauco.

\section{RESULTADOS}

\section{Análisis espectral del viento y de la corriente en la banda mareal}

El espectro de la componente este-oeste y norte-sur de la serie de viento mostró consistentemente la 
predominancia de una frecuencia típica de $0.042 \mathrm{cph}$ asociada a un período de $24 \mathrm{~h}$ con un nivel de confianza del $95 \%$ (Fig. 2a) y $80 \%$ (Fig. 2b), respectivamente. Esta frecuencia corresponde al proceso de brisa marina (Picarte et al., 1996). Para los otros peaks significativos asociados a la componente norte-sur no se tiene una explicación (Fig. 2a). El análisis de las componentes $u$ (este-oeste) y $v$ (norte-sur) de la corriente mareal mostró, en general, una frecuencia típica de $0,0805 \mathrm{cph}$ asociado a un período de $12.42 \mathrm{~h}$, correspondiente a un régimen mareal semidiurno (M2) (Fig. 3 y Fig. 4). Las excepciones corresponden a las densidades espectrales de la componente $u$ de la corriente en PP y $\mathrm{CG}$, las que no mostraron frecuencias significativas asociadas al período de $12.42 \mathrm{~h}$ (Fig. 3c, 3d y 3e).

\section{Análisis de la banda subinercial: Caracterización de eventos de surgencia en el Golfo de Arauco}

Durante los días en que se realizaron los cruceros hidrográficos $(5,9,14,22$ y 27 de marzo) y fueron registradas imágenes de TSM (10, 16, 22 y 27 de marzo) el viento promedio horario fue predominantemente SW (Fig. 5). En este mes se observó 4 períodos de viento $\mathrm{N}$ (11-13, 19-20, 23-24 y 30-31), siendo el primer período el más intenso (Fig. 6). Los valores del índice de
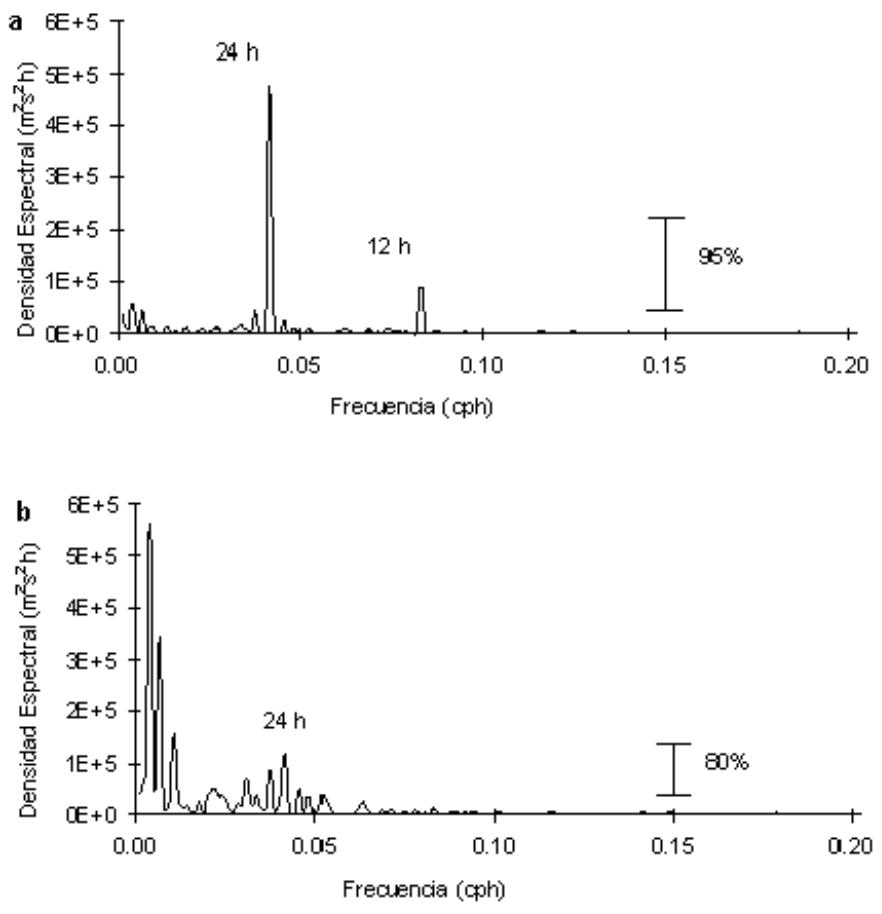

Figura 2. Densidad espectral de las componentes (a) este-oeste y (b) norte-sur de la velocidad del viento registrada en la estación meteorológica Carriel Sur.

Figure 2. Spectral density of the (a) east-west and (b) north-south component of the wind speed of data recorded by the Carriel Sur meteorological station. surgencia promedio (Is) mostraron períodos alternados de surgencia (Is • $\left.10 \mathrm{~m}^{3} \mathrm{~s}^{-1}\right)$ y relajación $\left(\mathrm{Is}<10 \mathrm{~m}^{3} \mathrm{~s}^{-1}\right)$ con valores no mayores a $40 \mathrm{~m}^{3} \mathrm{~s}^{-1}$ (Fig. 6). El límite impuesto para el índice de surgencia supone una velocidad vertical $\bullet 10 \mathrm{~m}$ por día, es decir, una velocidad $\bullet 10^{-4} \mathrm{~ms}^{-1}$. Este valor de ascenso vertical se asocia a un esfuerzo del viento del orden de $10^{-2} \mathrm{Nm}^{-2}$, lo que equivale a un Is de $10 \mathrm{~m}^{3} \mathrm{~s}^{-1}$. Basado en esta estimación los períodos de surgencia correspondieron a: 2-8, 14-18, 21-22 y 25-29 de marzo con Is promedio iguales a $17,18,13$ y $21 \mathrm{~m}^{3} \mathrm{~s}^{-1}$, respectivamente. Los períodos de relajación correspondieron a 9-13, 19-20, 23-24 y 29-30 de marzo con Is de $-11,-1,-11$ y $-3 \mathrm{~m}^{3} \mathrm{~s}^{-1}$, respectivamente.

\section{Desplazamiento residual en el Golfo}

Los diagramas de vector progresivo (DVP) evidenciaron un sistema que cambia desde una a dos capas de circulación dependiendo de los cambios de la dirección e intensidad del viento, de la influencia de la surgencia y del hundimiento en el área adyacente al Golfo. Durante períodos de surgencia y de hundimiento la dirección de la corriente en los estratos de superficie y subsuperficie presentó la misma dirección. Por ejemplo, los períodos de surgencia 7-9 y 25 26 de marzo, la dirección de la corriente en el costado oeste del Golfo (PD) en ambos estratos fue hacia el SE (sureste) mostrando una sola capa (Figs.7a y 7b). Durante el período de máximo viento $\mathrm{N}$ observado entre el 12 y 13 de marzo, la dirección de la corriente en el costado E (PP) fue hacia el SW en ambos estratos (Figs. 8a y 8b). Por el contrario, en períodos de transición de viento $\mathrm{S}$ a $\mathrm{N}$ o de $\mathrm{N}$ a $\mathrm{S}$, la dirección de la corriente en el estrato de superficie cambia de dirección o se debilita, mostrando la presencia de dos estratos con intensidades y direcciones disími-les. Esto es observado tanto en PD como en PP. Un ejemplo de esto último corresponde al período 14-17 de marzo, pe- 

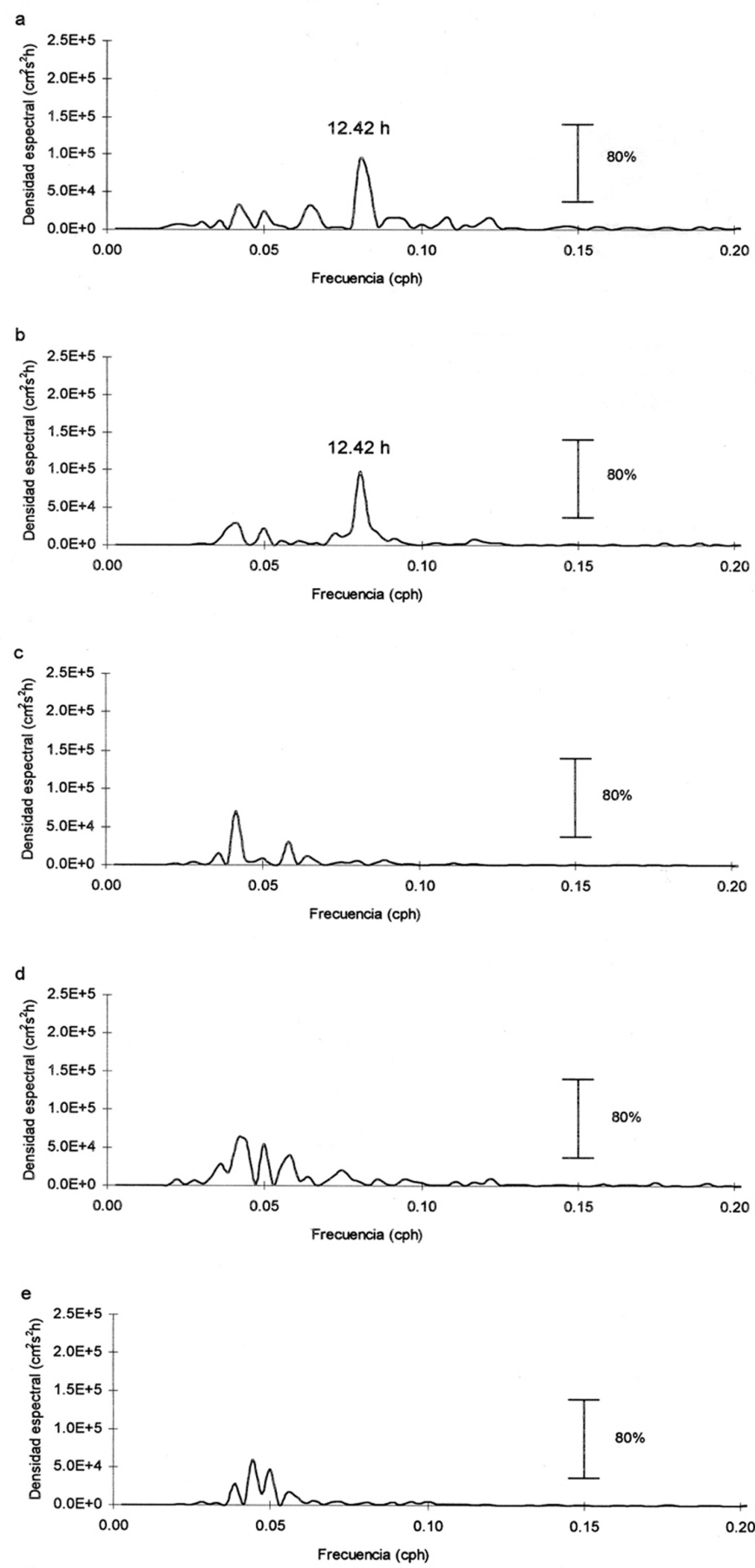

Figura 3. Densidad espectral de la componente u (este-oeste) de la corriente mareal de los correntómetros ubicados en (a) PD a $10 \mathrm{~m}$, (b) PD a $28 \mathrm{~m}$, (c) CG a $25 \mathrm{~m}$, (d) PP a $10 \mathrm{~m}$, (e) PP a $28 \mathrm{~m}$.

Figure 3. Spectral density of the east-west component (u) of the tidal current of data recorded for current meters located at (a) PD at $10 \mathrm{~m}$ depth, (b) PD at $28 \mathrm{~m}$ depth, (c) CG at 25m depth, (d) $P P$ at $10 \mathrm{~m}$ depth, (e) PP at $28 \mathrm{~m}$ depth. ríodo de transición de viento $\mathrm{N}$ a viento S-SW, donde la capa de superficie en PD cambia su dirección a el SE, mientras que el estrato de fondo permanece con una dirección hacia el NW mostrando la presencia de direcciones opuestas en ambos niveles de profundidad (Figs.7a y 7b). Las diferencias en la dirección e intensidad de la corriente tanto en el costado Oeste (correspondiente a PD), Este (correspondiente a PP) o en la cabeza del Golfo (CG, Fig. 8c) serán analizadas posteriormente para cada una de las situaciones características en un evento surgencia-relajación.

\section{Imágenes satelitales de TSM y desarrollo de un evento relajación- surgencia}

En la Figura 9 se muestra la variabilidad de la TSM durante los días 10, 16, 22 y 27 de marzo. Estos días correspondieron a períodos de surgencia a excepción del período de relajación del día 10 de marzo (Fig. 6). Las imágenes de TSM fueron relacionadas a la dirección e intensidad del viento y a los desplazamientos residuales de la corriente. Los eventos de surgencia y de relajación se interpretaron en función de los focos de surgencia adyacentes al Golfo y a la presencia de agua de baja temperatura en superficie $\left(11^{\circ} \mathrm{C}\right)$. A partir de esta información se plantea el siguiente modelo de desarrollo de un evento de Surgencia-Relajación.

\section{Debilitamiento del viento SW y fortalecimiento del viento $\mathrm{N}$}

Los días 9 y 10 se produjo un debilitamiento del viento S-SW (promedio diario de $1.67 \mathrm{~ms}^{-1}$ ) y el 11,12 y 13 de marzo se observó un predominio del viento N (Fig. 5). La circulación entre estos dos períodos cambió desde dos capas (una superficial antihoraria y otra subsuperficial horaria, días 9 y 10, Fig. 9a) a una sola capa de tendencia horaria (días 12 y 13 de marzo. La Tabla 1 resume la dirección residual de las corrientes asignándoles un sentido de rotación horaria o antihoraria cuando corresponde. La intensidad de la corriente se estableció en 

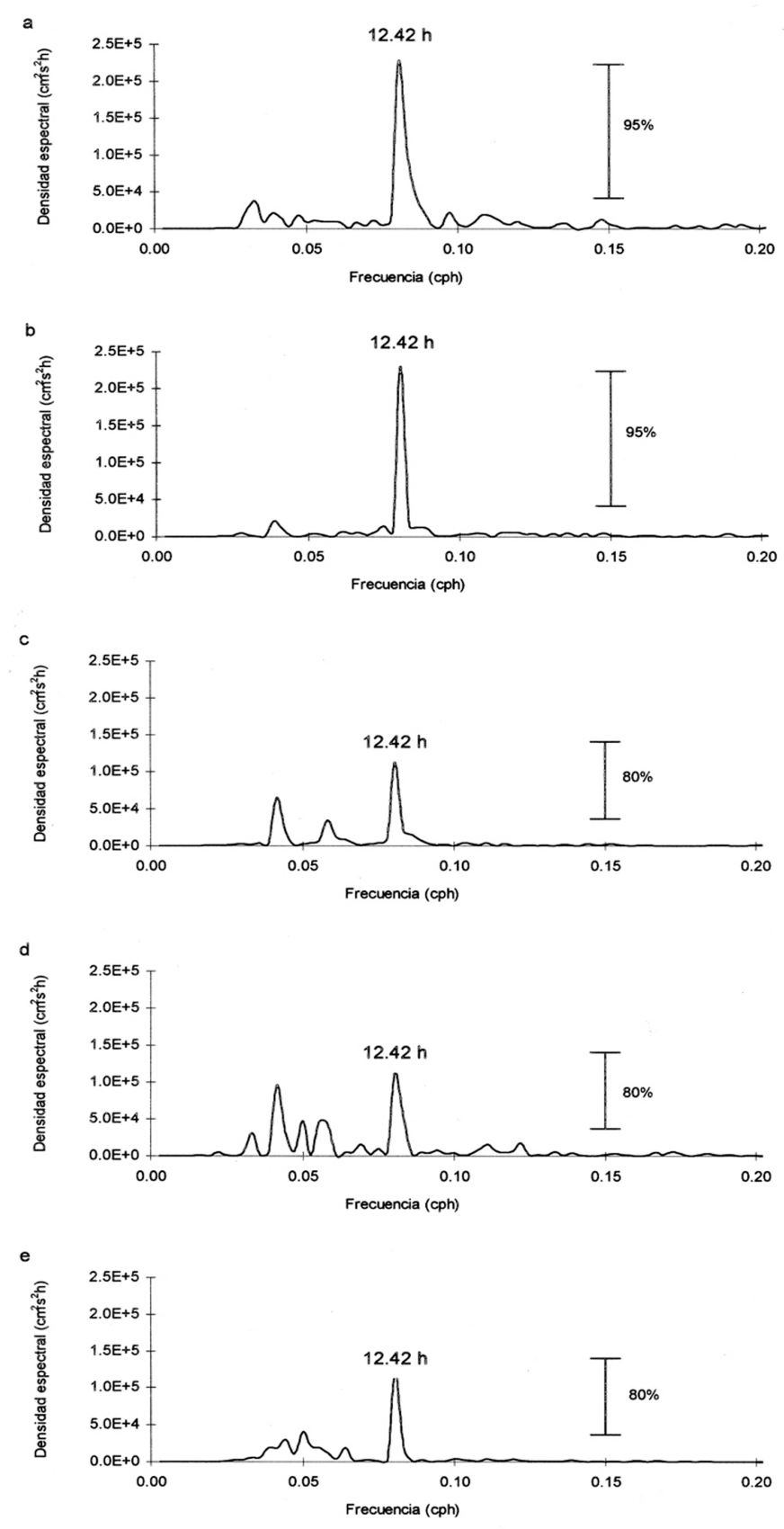

Figura 4. Densidad espectral de la componente $v$ (norte-sur) de la corriente mareal de los correntómetros ubicados en (a) Punta Delicada (PD) a $10 \mathrm{~m}$, (b) Punta Delicada (PD) a $28 \mathrm{~m}$, (c) Cabeza del Golfo (CG) a 25 m, (d) Punta Puchoco (PP) a 10 m y (e) Punta Puchoco (PP) a $28 \mathrm{~m}$.

Figure 4. Spectral density of the north-east component (v) of the tidal current of data recorded for current meters located at (a) Punta Delicada (PD) at 10 m depth, (b) Punta Delicada (PD) at 28 m depth, (c) Cabeza del Golfo (CG) at 25m depth, (d) Punta Puchoco (PP) at 10 m depth, (e) Punta Puchoco (PP) at 28 m depth. términos relativos. Las TSM no mostraron temperaturas menores a $13^{\circ} \mathrm{C}$ dentro o fuera del Golfo.

\section{Máximo hundimiento}

El día 13 de marzo se observó una capa con la máxima tendencia horaria del período que coincidió con el predominio de viento $\mathrm{N}$ (Tabla 1 ). A diferencia del período de máxima surgencia, este período se caracterizó por una corriente de salida de agua por la parte oeste del Golfo. Este flujo de salida mostró una intensificación en el nivel de fondo.

\section{Debilitamiento del viento $\mathbf{N}$ e inicio del viento $\mathrm{S}-\mathrm{SW}$}

Durante el período 14-17 de marzo se observó un debilitamiento del viento $\mathrm{N}$ y el fortalecimiento de viento S-SW (promedio diario de 3,26 ms ${ }^{-1}$, Fig. 5). La circulación al comienzo del período se caracterizó por la presencia de una sola capa horaria (Tabla 1), posteriormente la circulación mostró un sentido horario débil en superficie y un patrón horario de fondo (Fig. 9b) Sin embargo, hacia el final del período (17 de marzo) se produjo un fortalecimiento del viento S-SW el cual generó una circulación de dos capas (antihoraria en superficie y horaria en subsuperficie, Tabla 1). La TSM (día 16 de marzo, Fig. 9b) mostró en el centro del Golfo agua de $12^{\circ} \mathrm{C}$ y delgadas franjas de agua de $11^{\circ} \mathrm{C}$ restringidas hacia la costa Este del Golfo, al S de Punta Lavapié (37 $22^{\circ}$ S, $\left.73^{\circ} 40^{\prime} \mathrm{W}\right)$ y al N de Bahía de Concepción ( $\left.36^{\circ} 40^{\prime} \mathrm{S}, 72^{\circ} 57^{\prime} \mathrm{W}\right)$. Estos antecedentes podrían ser indicio de la gestación de un evento de surgencia a lo largo de esta zona costera.

\section{Incremento del viento S-SW y} desarrollo de surgencia en el área adyacente al Golfo

Los días 21 y 22 muestran el incremento del viento S-SW. Al comienzo del período la circulación no mostró un patrón claro en superficie mientras que en subsuperficie presentó una tenden- 
a

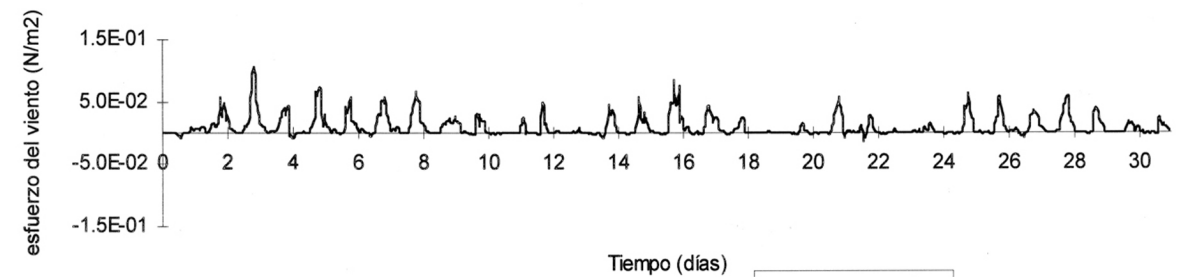

b

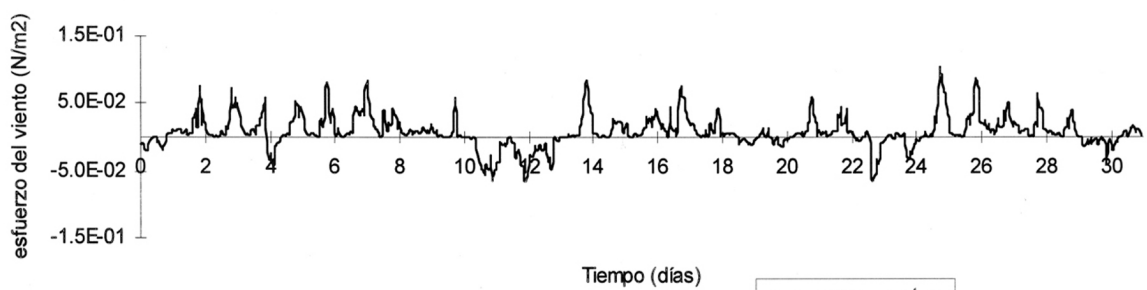

Figura 5. Esfuerzo del viento de datos registrados en la estación meteorológica Carriel Sur.

Figure 5. Wind stress from data recorded at the Carriel Sur meteorological station.

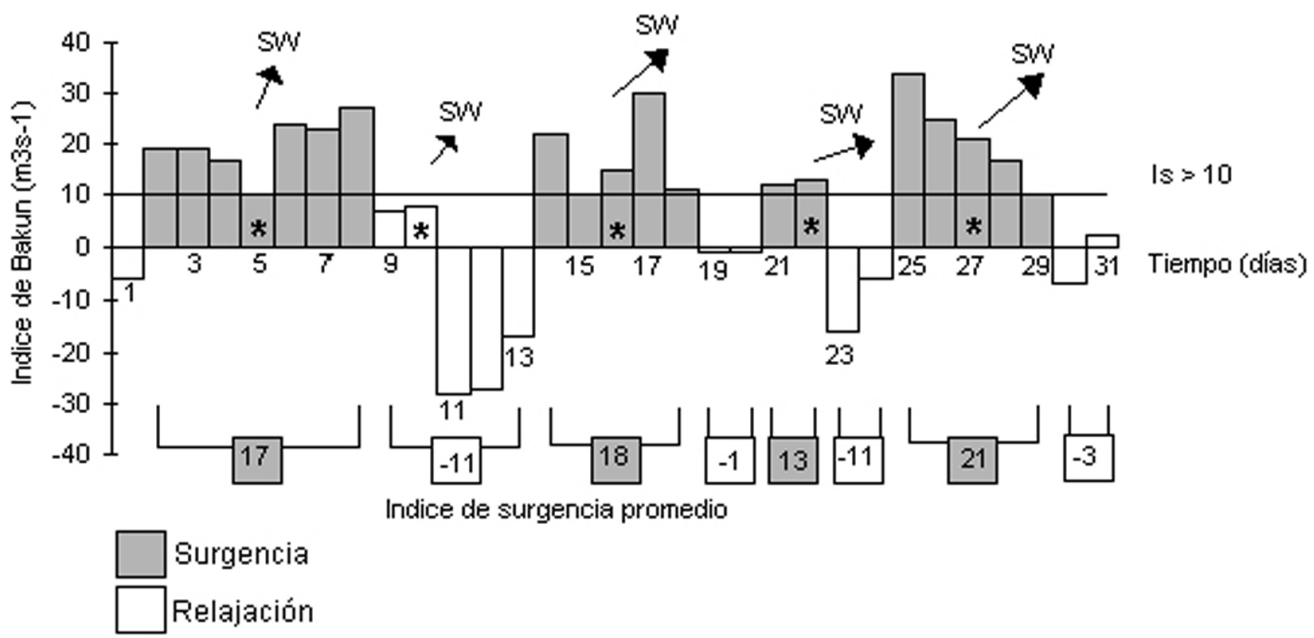

Figura 6. Indice de surgencia de Bakun para vientos, indicando la alternancia de eventos de surgencia y relajación registrados en la Estación Carriel Sur durante marzo de 1996 (* corresponde al día de registro de TSM).

Figure 6. Daily upwelling index for wind data, indicating the alternance of upwelling and relaxation events recorded at the Carriel Sur station during March of (indicates that SST image of the Arauco Gulf is available for the corresponding day). 
a

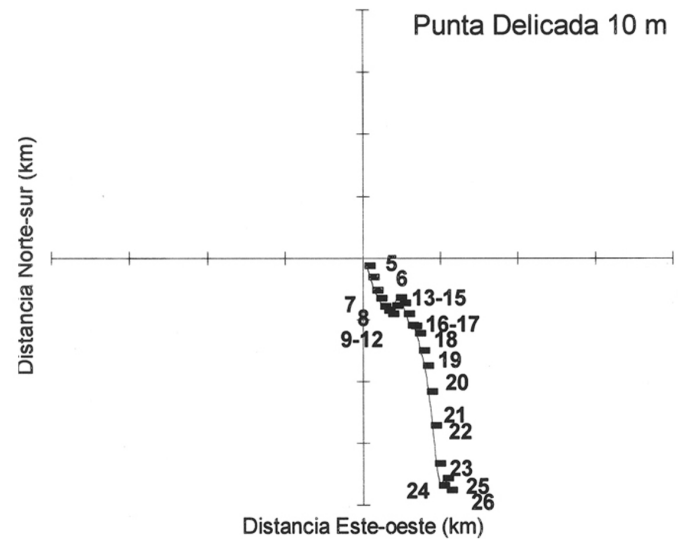

b

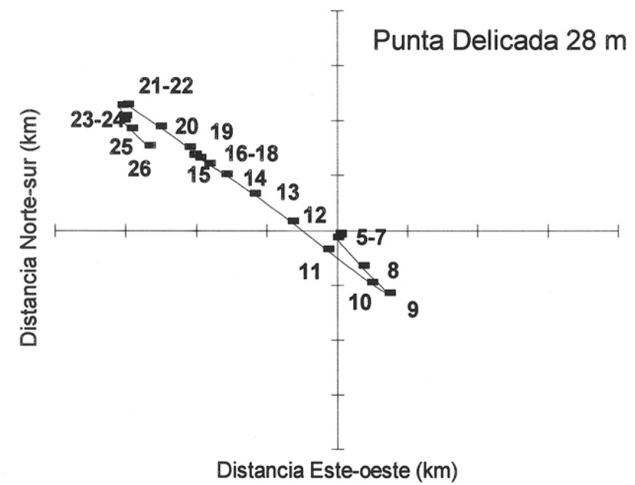

Figura 7. Diagrama del vector progresivo para los datos registrados por los correntómetros ubicados en (a) Punta Delicada (PD) a 10 m y (b) Punta Delicada (PD) a $28 \mathrm{~m}$.

Figure 7. Progressive vector diagram for 5 current meters installed at (a) Punta Delicada (PD) at $10 \mathrm{~m}$ of depth and (b) Punta Delicada at $28 \mathrm{~m}$ of depth.
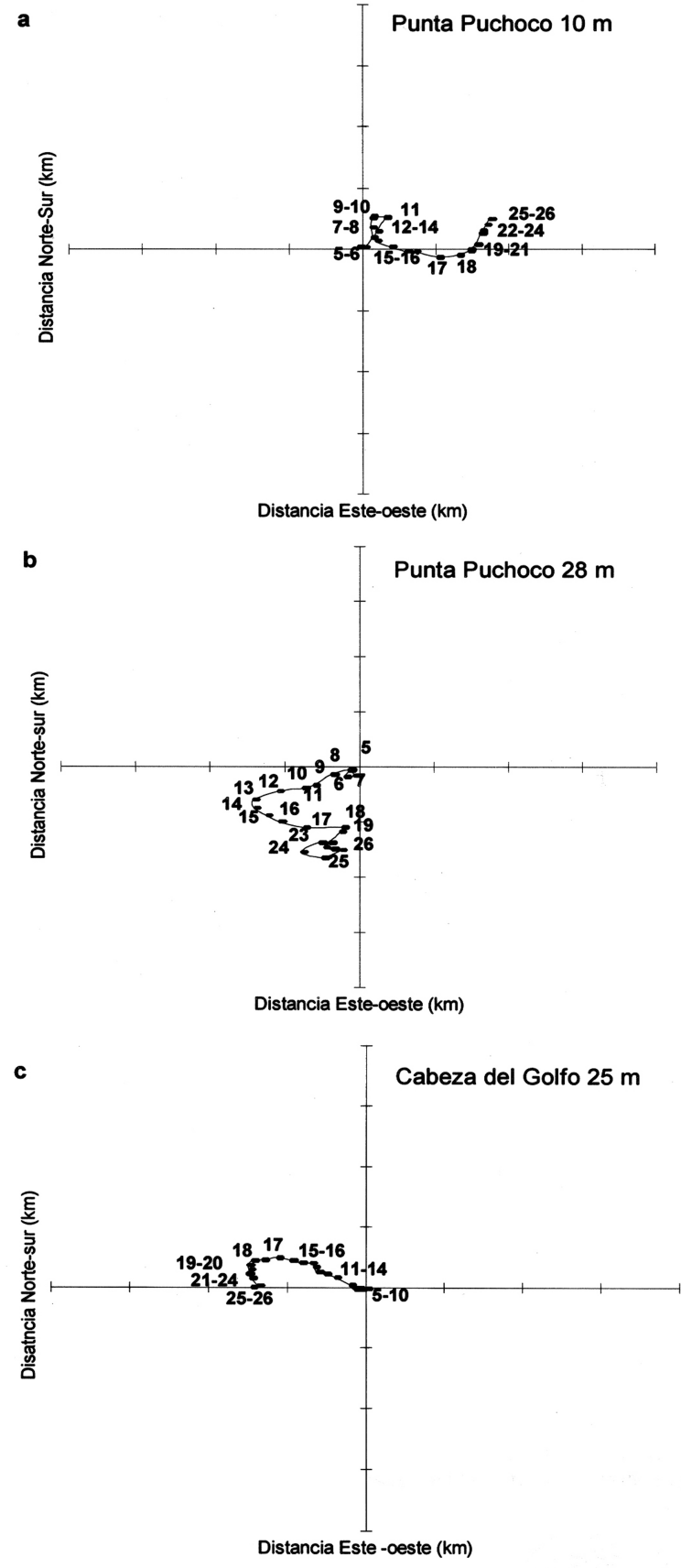

Figura 8. Diagrama del vector progresivo para los datos registrados por los correntómetros ubicados en (a) Punta Puchoco (PP) a 10 m, (b) Punta Puchoco a $28 \mathrm{~m}$ y (c) Cabeza del Golfo a $25 \mathrm{~m}$.

Figure 8. Progressive vector diagram for the current meters installed at (a) Punta Puchoco (PP) at 10 m depth, (b) Punta Puchoco at $28 \mathrm{~m}$ depth and (b) Cabeza del Golfo at $25 \mathrm{~m}$ depth. 
Tabla 1. Dirección e intensidad de la corriente en Punta Delicada (PD) y Punta Puchoco (PP) a 10 y 28 m y en Cabeza del Golfo (CG) a $25 \mathrm{~m}$. Cs es la circulación en superficie y Cf la circulación de fondo, las que pueden ser horaria (H), antihoraria (A) o difusa (D). La intensidad de la corriente puede ser intensa (I), moderada (M) o débil (D).

Table 1. Direction and intensity of currents at Punta Delicada (PD) and Punta Puchoco (PP) at 10 and $28 \mathrm{~m}$ of depth, and at Cabeza del Golfo (CG) at $\mathbf{2 5} \mathbf{~ m}$ of depth. Cs and Cf are the surface and subsurface circulation, respectively, that can be clockwise $(H)$, anticlockwise $(A)$ or diffuse $(D)$. The strength of the current can be intense (I), moderate (M) or weak (D).

\begin{tabular}{|c|c|c|c|c|c|c|c|}
\hline \multicolumn{6}{|c|}{ Dirección e intensidad de los correntómetros } & \multicolumn{2}{|c|}{ Tipo de circulación } \\
\hline Día & PD10 & PD28 & PP10 & PP28 & CG25 & Cs & $\mathbf{C f}$ \\
\hline 5 & SE (I) & SE (M) & NW (M) & SW (M) & SW (M) & A débil & A \\
\hline 7 & SE (I) & SW (D) & $\mathrm{NE}(\mathrm{M})$ & SE (D) & NW (D) & A & Di \\
\hline 8 & SW (M) & SE (I) & NW (M) & $\mathrm{NW}$ (D) & SW (D) & A & Di \\
\hline 9 & $\mathrm{SE}(\mathrm{M})$ & SE (I) & $\mathrm{NE}(\mathrm{M})$ & SW (M) & NW (D) & A & $\mathrm{H}$ \\
\hline 10 & SE (D) & NW (I) & NW (D) & $\mathrm{SW}(\mathrm{M})$ & NW (D) & A & $\mathrm{H}$ \\
\hline 11 & SE (D) & NW (I) & $\mathrm{NE}$ (D) & SW (D) & NW (M) & A & $\mathrm{H}$ \\
\hline 12 & NW (M) & NW (I) & SW (I) & SW (M) & NW (M) & $\mathrm{H}$ & $\mathrm{H}$ \\
\hline 13 & NW (M) & NW (I) & SW (M) & SW (M) & NW (D) & $\mathrm{H}$ & $\mathrm{H}$ \\
\hline 14 & $\mathrm{SE}(\mathrm{M})$ & $\mathrm{NW}(\mathrm{I})$ & SE (M) & SE (M) & NW (D) & $\mathrm{H}$ & $\mathrm{H}$ \\
\hline 15 & SE (M) & NW (I) & SE (M) & SE (M) & NW (D) & $\mathrm{H}$ & $\mathrm{H}$ \\
\hline 16 & $\mathrm{SE}(\mathrm{M})$ & NW (M) & $\mathrm{NE}(\mathrm{D})$ & $\mathrm{SE}(\mathrm{M})$ & NW (D) & H débil & $\mathrm{H}$ \\
\hline 17 & SE (I) & NW (D) & SE (I) & SE (M) & NW (M) & A & $\mathrm{H}$ \\
\hline 22 & SE (I) & SW (D) & NE (D) & SE (D) & SE (D) & A & A débil \\
\hline 26 & SE (I) & SE (I) & $\mathrm{NE}(\mathrm{M})$ & NE (I) & SE (D) & A & A \\
\hline
\end{tabular}

cia horaria (Tabla 1). El 22 de marzo correspondió a un período de fortalecimiento del viento S-SW (promedio diario de 2,84 $\mathrm{ms}^{-1}$ ) mostrando una tendencia antihoraria en superficie y antihoraria más débil en subsuperficie (Fig. 9c). Estos cambios en la dirección de la corriente parecen indicar el comienzo de un patrón antihorario asociado al inicio del desarrollo de surgencia adyacente al Golfo. A diferencia del período precedente la surgencia se caracteriza por franjas de agua de $11^{\circ} \mathrm{C}$ al oeste de la ${ }^{2}$ boca chica ${ }^{2}$ y asociada a Punta Lavapié (Fig. 9c).

\section{Máxima surgencia en el área adyacente al Golfo}

El 27 de marzo correspondió a un período de surgencia con predominio de viento SW (promedio diario $3,77 \mathrm{~ms}^{-1}$, Fig. 9d). Tanto dentro como fuera del Golfo la temperatura superficial fue dominada por agua $-11^{\circ} \mathrm{C}$. Durante el máximo de surgencia se observó una intensificación en la velocidad de la corriente en todos los correntómetros. Se observó sólo una capa con tendencia antihoraria (Tabla 1) y una notable entrada de agua hacia el Golfo por la parte oeste, con una clara intensificación de la corriente en el fondo (Fig. 9d).

\section{DISCUSIÓN Y CONCLUSIONES}

En este estudio se caracterizan los patrones de circulación en el interior del Golfo de Arauco que están asociados a períodos de intensificación y relajación de la surgencia costera durante un período de transición verano-otoño. Los resultados concuerdan en gran medida con la información previa (Alarcón, 1970; Bernal et al., 1986; Djurfeldt, 1989; Urrutia, 1993; Sobarzo et al., 1993). Los forzantes que regulan la dinámica del sistema son, principalmente, la marea y el viento. El régimen mareal semidiurno concuerda con los resultados entregados por Blanco (1984) realizados en la plataforma continental de Talcahuano. El análisis del viento y su espectro fueron consistentes con los resultados obtenidos por Saavedra (1980) y Djurfeldt (1989), en el que se observa un predominio de viento SW y esporádicas 

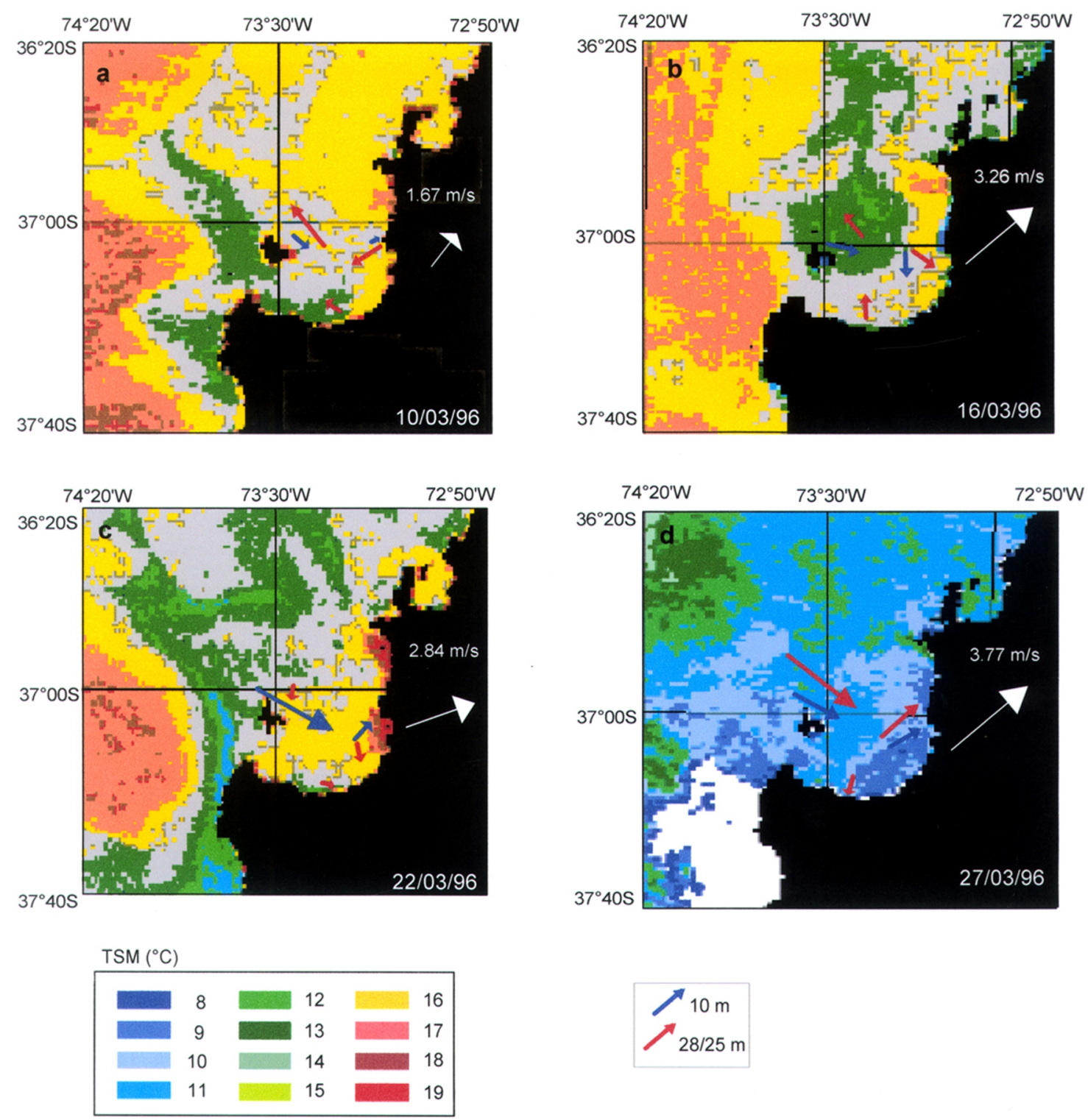

Figura 9. Imágenes satelitales de la temperatura superficial del mar para marzo de 1996, indicando los vectores de velocidad del viento (flecha blanca) y de la corriente en superficie (flecha azul) y subsuperficie (flecha roja). Las imágenes fueron obtenidas los días (a) 10, (b) 16, (c) 22 y (d) 27 de marzo de 1996.

Figure 9. Satellite images of SST in the Arauco Gulf during March 1997, indicating the velocity vectors of wind (white arrow), and surface (blue arrow) and subsurface (red arrow) currents. The images were registered on (a) 10, (b) 16, (c) 22 and (d) 27 of March. 
interrupciones de viento $\mathrm{N}$, con una fuerte señal diaria asociada al período de estudio. Esta fluctuación diaria, denominada brisa marina, fue identificada Ruiz (1994), Herrera (1994) y Picarte et al. (1996).

Del análisis de los datos se concluye que la predominancia de viento S-SW generó una circulación antihoraria, mientras que el predominio de viento $\mathrm{N}$ provocó una circulación horaria. La transición desde una situación de viento $\mathrm{N}$ a S-SW generó un debilitamiento de la capa superficial y un cambio en su sentido de rotación desde horaria a antihoraria. Probablemente, en períodos de transición a viento $\mathrm{N}$ la circulación de la corriente mostrará un sistema de doble capa: superficial antihoraria y subsuperficial horaria. Durante el predominio de viento S-SW, se intensifica la entrada de agua por el lado oeste del golfo en superficie y subsuperficie. A medida que se desarrolla el proceso la velocidad de la capa subsuperficial se hace mayor ingresando agua por el costado oeste y saliendo por el costado este del Golfo. Durante la relajación de la surgencia, la entrada de agua por el lado oeste del Golfo se debilita en toda la columna de agua llegando, incluso, a una inversión de la dirección de la corriente en subsuperficie. Cuando el viento $\mathrm{N}$ se intensifica la salida de agua se fortalece por el costado oeste del Golfo. Durante el máximo desarrollo de la surgencia, así como durante máximo hundimiento, se propone que la dinámica en el interior del Golfo está gobernada por una sola capa de circulación.

A partir de los datos analizados se propone a continuación un modelo conceptual para explicar la dinámica de las corrientes asociadas a eventos de intensificación y relajación de la surgencia. En este modelo se caracterizan los patrones de circulación al interior del Golfo durante un período de surgenciarelajación. Se indican dos situaciones extremas (surgencia y hundimiento) junto a dos períodos de transición entre ellas. El ciclo completo se acompaña con una descripción del viento predominante (Figura 10).

(1) El viento $\mathrm{N}$ genera procesos de hundimiento de aguas en la plataforma lo que se refleja en una entrada de agua por el costado este del Golfo y por un flujo de salida de agua por su costado oeste (más intenso en el fondo). Esto genera una circulación horaria de una sola capa en el interior del Golfo.

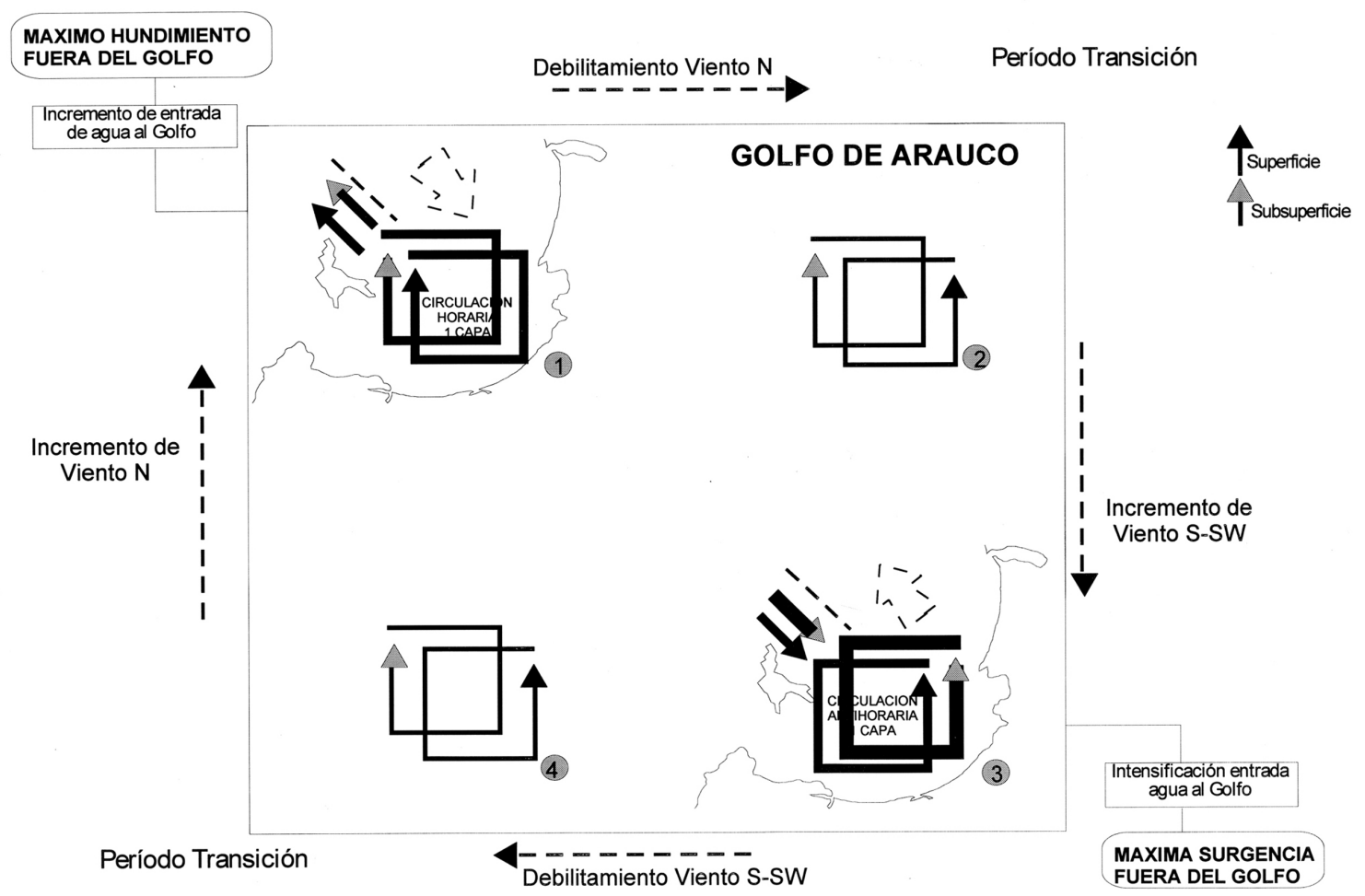

Figura 10. Esquema conceptual del proceso Surgencia-Relajación en un período de transición verano-otoño. Figure 10. Conceptual scheme of the Upwelling-Relaxation process in a summer-autumn transition. 
(2) Transición viento $\mathrm{N}$ a viento $\mathrm{S}$. Posterior al viento $\mathrm{N}$ y debido al incremento del viento $\mathrm{S}$ sólo la capa superficial cambia su sentido de rotación a antihorario. Esta situación de transición provoca dos capas de circulación con direcciones opuestas y en ambos costados del Golfo.

(3) La intensificación del viento SW provoca surgencia costera lo cual produce un ingreso de agua por el costado oeste del Golfo y una salida por su costado este. En este caso la circulación dentro del Golfo presenta sólo una capa de circulación con sentido antihorario.

(4) Transición viento $S$ a viento N. Posterior al viento $\mathrm{SE}$ e incremento del viento $\mathrm{N}$, la circulación superficial presenta un giro antihorario superficial y un giro horario en subsuperficie.

El presente modelo constituye una aproximación conceptual que tiende a definir los patrones de circulación en el interior del Golfo. Demuestra que estos patrones están fuertemente ligados a los procesos de surgencia y hundimiento que ocurren adyacentes al Golfo. La validación de este tipo de modelos requerirá de mediciones temporales más largas de corrientes y de la utilización de técnicas de modelación numérica.

\section{AGRADECIMIENTOS}

Este estudio fue financiado por el proyecto FoNDECYT 39500-24 adjudicado al Dr. Leonardo Castro. Financiamiento parcial durante la generación del manuscrito fue otorgado por FondAP-Humboldt a los autores Leonardo Castro y Dante Figueroa. Se agradece la colaboración del Centro EULA de la Universidad de Concepción por la instalación del equipo de correntometría (a cargo del Sr. Jaime Henríquez) y al Laboratorio del Programa Multidisciplinario de Percepción Remota (PMPR) de la Universidad de Concepción por el procesamiento de las imágenes satelitales de temperatura superficial del mar (TSM).

\section{REFERENCIAS}

Alarcón, E. 1970. Descripción oceanográfica preliminar para el Golfo de Arauco. Instituto de Fomento Pesquero, Boletín Científico, 13, 35 pp.
Ahumada, R. y L. Chuecas. 1979. Algunas características hidrográficas de la Bahía de Concepción $\left(36^{\circ} 40^{\prime} \mathrm{S} ; 73^{\circ} 02^{\prime} \mathrm{W}\right)$ y áreas adyacentes, Gayana Misc., 8: 1-56.

Arcos, D. y M.A. Salamanca. 1984. Distribución de clorofila y condiciones oceanográficas superficiales frente a Chile central (Latitudes $32^{\circ} \mathrm{S}-38^{\circ} \mathrm{S}$, Febrero 1982). Biol. Pesq., 13: 5-14.

Arcos, D., S. Núñez, L. Castro y N. Navarro. 1987. Variabilidad vertical de la clorofila en un área de surgencia frente a Chile central. Invest. Pesq., Valparaíso, 34: 47-55.

Arcos, D., S. Núñez y A. Acuña. 1996. Variabilidad de pequeña escala en la zona nerítica del sistema de surgencia de Talcahuano (Chile Central): Identificación y dinámica de áreas de retención larval. Gayana Oceanol., 4(1): 21-58.

Bakun, A. 1978. Daily and weekly upwelling indices, west coast of North America, 1967-73. U.S. Commer. NOAA Tech.Rep. NMFS-SSRS-693, pp. 113.

Bernal, A., P.R. Ahumada, H. González, V.A. Troncoso, G. Herrera, V. Martínez y L. Castro. 1986. Formación /destrucción de capas en un área costera altamente productiva. Informe de avance proyecto DIUC, Pontificia Universidad Católica de Chile, Talcahuano.

Blanco, J.L. 1984. Características de la circulación sobre la plataforma continental de Talcahuano. Tesis de Oceanógrafo. Escuela de Ciencias del Mar. Universidad Católica de Valparaíso, 42 pp.

Castillo, G., H. Muñoz, H. González y P. Bernal, 1991. Daily analysis of abundance and size variability of fish larvae in relation to oceanic water intrusions in coastal areas. Biol. Pesq., 20: 21-35.

Castro, L., R. Quiñones, H. Arancibia, R. Roa, M. Sobarzo y M. Retamal. 1997. Areas de desove de anchoveta y sardina común en la zona central de Chile. Informe Final, Proyecto Fondo de Investigación Pesquera, 96-11, 114 pp.

Djurfeldt, L. 1989. Circulation and mixing in the coastal upwelling embayment; Gulf of Arauco, Chile. Cont. Shelf Res., 9: 1003-1016.

Herrera, M.A. 1994. La brisa marina superficial en la octava región: un análisis temporal y espacial. Tesis de Biólogo Marino. Departamento de Oceanografía. Universidad de Concepción, 58 pp. 
Jenkins, G.M. y D.G. Watts. 1969. Spectral analysis and its applications. Holden-Day, $525 \mathrm{pp}$.

Krause, G. (ed.). 1984. Practical course in physical oceanography. University of San Carlos. Philippines, $49 \mathrm{pp}$.

Pizarro, O. 1991. Propagación y forzamiento de perturbaciones de baja frecuencia del nivel del mar en la costa norte de Chile. Tesis de Oceanógrafo. Escuela de Ciencias del Mar. Universidad Católica de Valparaíso., 105 pp.

Picarte, C., M. Contreras y A. Urrutia. 1996. Ondelette: una aplicación para caracterizar la brisa marina superficial en el Golfo de Arauco (Chile). Rev, Biol. Mar., Valparaíso, 31(2): 123-138.

Ruiz, V.R. 1994. La brisa marina superficial en la Octava Región. Tesis de Magister en Física. Escuela de Graduados. Universidad de Concepción, Chile.

Saavedra, N. 1980. La presión y dirección del viento en Concepción. Tralka, 1(2): 153-162.

Sobarzo, M. 1993. Caracterización de la circulación de Bahía Concepción, Chile: un análisis temporal en el dominio de la frecuencia. Tesis de Magister en Ciencias mención Oceanografía. Universidad de Concepción, 128 pp.

Sobarzo, M., E. Sansone, A. De Maio, D. Arcos, M. Salamanca y J. Henríquez. 1993. Oceanografía física del Golfo de Arauco. Variabilidad espacio temporal de la estructura hidrográfica de las aguas del Golfo de Arauco. Primera parte. F. Faranda y O. Parra (ed.). Universidad de Concepción, Serie Monografías Científicas, 4, 152 pp.
Sobarzo, M. 1994. Hydrographic and dynamic characteristics of the Arauco Gulf and San Vicente Bay. En: Management of water resources of a river basin and adjacent marine coastal area. Application of the EULA model in Chile on the Biobio river basin-gulf of Arauco- San Vicente Bay system. F. Faranda, R. Frache, O. Parra and P. Povero (eds.). International Colloquium, Paris, 224 pp.

Thompson, R. 1983. Low-pass filters to suppress inertial and tidal frequencies. J. Phys. Oceanogr., 13: 1077-1083.

Urrutia, A. 1993. Análisis del comportamiento de la pluma del río Biobío en base a un estudio de sus campos de temperatura, salinidad, densidad y de movimiento. Tesis de Doctorado en Ciencias Ambientales, EULA, Universidad de Concepción, 195 pp.

Walters, R.A. y C. Heston, 1982. Removing tidal period variations for time series data using lowpass digital filters. J. Phys. Oceanogr., 12: 112-115.

Wu, J. 1982. Wind-stress coefficients over sea surface from breeze to huricane. J. Geophys. Res., 82: 9704-9706.

Yáñez, E., V. Catasti, M.A. Barbieri y G. Böhm. 1996. Relaciones entre la distribución de recursos pelágicos pequeños y la temperatura superficial del mar registrados con satélites NOAA en la zona central de Chile. Invest. Mar., Valparaíso, 24: 107122. 
